\title{
A new heuristic approach to deal with discrete variables in optimal power flow computations
}

\author{
Florin Capitanescu and Louis Wehenkel, Member, IEEE
}

\begin{abstract}
This paper proposes a new heuristic approach to deal with discrete variables in an optimal power flow (OPF). This approach relies on the first order sensitivity of the objective and inequality constraints with respect to the discrete variables. The impact of a discrete variable change on the objective and inequality constraints is aggregated into a merit function. The proposed approach searches iteratively for better discrete variable settings as long as the problem solution can be improved. We provide numerical results with the proposed approach on four test systems up to 1203 buses and for the OPF problem of active power loss minimization.
\end{abstract}

Index Terms-mixed integer nonlinear programming, nonlinear programming, optimal power flow

\section{INTRODUCTION}

$\mathbf{T}$ HE Optimal Power Flow (OPF) problem [1], [2] is a non-convex, large-scale, nonlinear programming problem with both continuous and discrete variables, in brief a mixed-integer nonlinear programming (MINLP) problem. MINLP problems belong to the category of very difficult (NPcomplete) optimization problems. The methods for solving MINLP can be roughly classified into deterministic and nondeterministic search. The former class includes innovative approaches and related techniques taken and extended from mixed integer linear programs (MILP), such as: branch and bound [3], [4], outer approximation [5], [6], generalized Benders decomposition [7], [8], interior point cutting plane [9], and extended cutting plane [10]. The latter class encompasses global optimization techniques such as: genetic algorithms, simulated annealing, tabu search, etc. All mentioned MINLP solution methods share the same drawback that they are nonpolynomial and hence present very poor scalability and prohibitive computational times, especially for large-scale problems. Besides, they only guarantee global optimality under feasible domain convexity assumptions with respect to the continuous variables.

Obviously, with the presently available computational tools, the above mentioned MINLP methods are still inappropriate for large-scale OPF applications, especially in the context of real-time operation. In fact, due to the time constraints, in the context of OPF computations in the framework of operational planning and especially real-time the main aim is to quickly find a near-optimal feasible solution while adequately handling the discrete variables.

The efficient handling of discrete variables in the OPF has been recognized as a challenging problem and has received

The authors are with the Department of Electrical Engineering and Computer Science, University of Liège, B4000 Liège, Belgium (e-mail: capitane@montefiore.ulg.ac.be; 1.wehenkel@ulg.ac.be). significant attention since the late 80's [11]-[13]. Meanwhile a large spectrum of approaches have been proposed to deal with discrete variables, e.g. simple heuristics [12], [15], penalty functions within NLP of LP solvers [16]-[18], ordinal optimization [19], recursive mixed-integer linear programming [20], interior point cutting plane [21], global optimization methods [22]-[25], etc.

The simplest approach for handling discrete variables is based on the rounding-off strategy [12]. In this technique, the OPF relaxation is first solved by treating all variables as continuous. Next, at the optimal solution, the discrete variables are rounded-off to their nearest discrete value. Finally, the discrete variables are frozen and the continuous variables are determined either by re-running the OPF, or by a classical power flow program. To reduce the sharp effect of roundingoff all discrete variables at once, a progressive rounding-off of discrete variables has been proposed [15]. In this approach at each iteration only a subset of discrete variables which are sufficiently close to a discrete value are rounded-off, the remaining variables (treated as continuous) being then reoptimized. It is largely agreed that the round-off technique is generally suitable for discrete variables with small steps (e.g., load tap changer (LTC) transformer ratio and phase shifter angle) but requires some caution for discrete variables with larger steps (e.g., shunt compensation banks, network switching) [12], [14], [16]. However, the round-off approaches act "blindly" since they do not look at the discretization effect on either the objective or the inequality constraints, suffering in consequence from two drawbacks: (i) the solution feasibility is not guaranteed while no method to restore feasibility is proposed, and (ii) the objective value may be unacceptably deteriorated.

Another class of heuristic approaches consists in handling discrete variables by means of penalty functions in NLP solvers such as the active-set Newton method [16] or the interior point method (IPM) [17]. The former approach uses several heuristic rules to drive the discrete variables to their discrete values, while nowadays the Newton method is seen as slightly less efficient than other NLP methods (e.g., interior point, sequential quadratic programming, etc.). The latter approach provides very good results, comparable with the performances of IPM solution of OPF relaxation. However, this approach uses very small steps for shunt banks (e.g., maximum 6 Mvar), whereas in real-life these steps are often significantly larger (e.g., up to 30-40 Mvar). Our experience with this approach shows that the convergence is much slower when considering larger steps, while sometimes the approach even experiences convergence problems. 
An interesting approach, very well suited for operational planning and even real-time applications, is proposed in [19]. It uses first a continuous OPF computation. Then it reduces the search space of discrete variables by keeping only the two neighbouring discrete values to the continuous solution. Finally, the OPF is re-run only for some combinations of discrete values, with an estimated better impact on the objective, the latter being selected by means of ordinal optimization theory. However, this approach does not address the problem of infeasibility caused by the discretization, in as much as the search space of discrete variables values is reduced to two discrete values per variable.

Last but not least the OPF problem has received a particular attention from the prespective of global optimization methods, let us quote non-exhaustively: genetic algorithms [22], [23], simulate annealing [24], tabu search [25], etc., or hybrid approaches coupling genetic algorithms and local search NLP solvers [26]. However, these techniques still remain heavy in terms of CPU times especially for large-scale systems.

In this paper we propose a new heuristic approach for handling efficiently discrete variables in the OPF problem. This approach relies on first order sensitivity of the objective and of the inequality constraints with respect to discrete variables. It looks iteratively for a better configuration of discrete variables based on their ability to improve the objective function and remove inequality constraints violation.

The rest of the paper is organized as follows. Section II recalls the general OPF formulation. Then, Section III presents the proposed sensitivity-based approach to deal with discrete variables. Section IV offers numerical results with the proposed approach and Section V concludes.

\section{The Optimal Power Flow PROBlem}

The classical OPF problem can be written as follows:

$$
\begin{array}{rl}
\min _{\mathbf{x}, \mathbf{u}_{c}, \mathbf{u}_{d}} & f\left(\mathbf{x}, \mathbf{u}_{c}, \mathbf{u}_{d}\right) \\
\text { s.t. } & \mathbf{g}\left(\mathbf{x}, \mathbf{u}_{c}, \mathbf{u}_{d}\right)=\mathbf{0} \\
& \mathbf{h}\left(\mathbf{x}, \mathbf{u}_{c}, \mathbf{u}_{d}\right) \leq \mathbf{0} \\
& \underline{\mathbf{u}}_{c} \leq \mathbf{u}_{c} \leq \overline{\mathbf{u}}_{c}, \mathbf{u}_{c} \in R^{n_{c}} \\
\mathbf{u}_{d} & =\left[u_{d 1} \ldots u_{d i} \ldots u_{d n_{d}}\right]^{T} \\
\mathbf{u}_{d i} & \in\left\{u_{d i}^{1}, \ldots, u_{d i}^{j}, \ldots, u_{d i}^{p(i)}\right\}, \forall i=1, \ldots, n_{d}
\end{array}
$$

where $\mathbf{x}$ is the vector of state variables (i.e., real and imaginary part of voltage at all buses), $\mathbf{u}_{c}$ is an $n_{c}$ dimensional vector of continuous control variables (e.g., generators active power, generators voltage (when controllable), load curtailment controls, etc.) and $\underline{\mathbf{u}}_{c}$ (resp. $\overline{\mathbf{u}}_{c}$ ) is its corresponding vector of lower (resp. upper) bounds, $\mathbf{u}_{d}$ is an $n_{d}$ dimensional vector of discrete control variables (e.g., LTC transformer ratios, shunt element reactances, phase shifters angle, etc.) and for the $i$-th discrete variable $u_{d i}^{j}$ is its $j$-th discrete value and $p(i)$ is its number of discrete positions, $f(\cdot)$ is the objective function, $\mathbf{g}(\cdot)$ and $\mathbf{h}(\cdot)$ are vectors of functions which model equality and inequality constraints. Equality constraints (2) are essentially the AC bus power flow equations, inequality constraints (3) refer to operational limits (e.g., branch currents and voltage magnitudes), inequality constraints (4) refer to physical limits of equipments (e.g., bounds on: generators active/reactive powers, load curtailment controls, etc.) while constraints (6) express that discrete variables $\mathbf{u}_{d}$ can take only discrete values.

A given configuration of the discrete variables $\mathbf{u}_{d}^{s}$ is called feasible if the continuous OPF problem (1-4) in which the discrete variables are fixed at the values specified by this configuration $\left(\mathbf{u}_{d}=\mathbf{u}_{d}^{s}\right)$ is feasible. Otherwise, it is called infeasible.

An enumeration approach applied to the OPF problem (1-6) requires the solution of $\prod_{i=1}^{n_{d}} p(i)$ continuous OPF problems. Assuming that all discrete variables have the same number $p$ of steps, the number of continuous OPF problems to solve is $p^{n_{d}}$ (e.g., for $p=10$ and $n_{d}=20$ one has $10^{20}$ possible configurations of the discrete variables). Therefore, in large scale systems with a large number of discrete variables, the exact solution of the MINLP OPF problem (1-6) is generally intractable by classical MINLP approaches.

\section{THE PROPOSED SENSITIVITY-BASED APPROACH}

\section{A. The underlying idea of the proposed approach}

The underlying idea of the proposed approach is to set values of discrete variables in a greedy fashion by assessing their ability to improve the objective and to remove inequality constraints violation. The first approach to come to mind in order to achieve this goal is based on finite differences. In this approach each discrete variable is moved to a new discrete position, a power flow (PF) program is run, and at its solution the variation of the objective and inequality constraints due to variable change is computed. Another approach consists in moving a discrete variables to a new discrete position and solve an OPF where discrete variables are frozen and only continuous variables are taken as control variables. If feasible, the solution of this OPF provides the shift in the objective due to discrete variable change while satisfying all constraints. These two approaches are accurate but very time consuming, especially the one based on OPF. Also, it is difficult to foresee whether the simultaneous changes in several discrete variables will be modeled with sufficient accuracy by superposing the effect of changing one discrete variable at the time.

In order to speed up computations while preserving sufficient accuracy of results we use instead an approach based on first order analytical sensitivities. Since sensitivities validity is ensured only for small variations around the operating point where they are derived, we restrict the discrete variables changes to a step (up or down) per iteration.

\section{B. Estimating the impact of discrete variable changes on the objective function and the inequality constraints}

We explain hereafter the sensitivity-based procedure that we use in order to choose control variables settings.

Let $\left(\mathbf{x}, \mathbf{u}_{c}, \mathbf{u}_{d}\right)$ describe a virtual operating point of the system, stemming from an OPF or a PF computation. Let us denote by $j_{i}\left(\forall i=1, \ldots, n_{d}\right)$ the current position of discrete variable $u_{d i}$. 
For an equilibrium point of the system there exist in the literature well known analytical formulas to compute first order sensitivities of some quantity of interest with respect to control variables [2], [27]. This computation may be performed at the solution of an OPF or a PF.

The key information of our approach is the sensitivity of the objective function and inequality constraints with respect to discrete variables $\mathbf{u}_{d}$ changes, which we denote by $\mathbf{S}_{\mathbf{u}_{d}}^{f}$ and $\mathbf{S}_{\mathbf{u}_{d}}^{\mathbf{h}}$. According to [2] these sensitivities take on the form:

$$
\begin{gathered}
\mathbf{S}_{\mathbf{u}_{d}}^{f}=\frac{\partial f}{\partial \mathbf{u}_{d}}-\left(\frac{\partial \mathbf{g}}{\partial \mathbf{u}_{d}}\right)^{T}\left[\left(\frac{\partial \mathbf{g}}{\partial \mathbf{x}}\right)^{T}\right]^{-1} \frac{\partial f}{\partial \mathbf{x}} \\
\mathbf{S}_{\mathbf{u}_{d}}^{\mathbf{h}}=\frac{\partial \mathbf{h}}{\partial \mathbf{u}_{d}}-\frac{\partial \mathbf{h}}{\partial \mathbf{x}}\left(\frac{\partial \mathbf{g}}{\partial \mathbf{x}}\right)^{-1} \frac{\partial \mathbf{g}}{\partial \mathbf{u}_{d}}
\end{gathered}
$$

Note that, if the point where the sensitivities are derived is an OPF optimum, the terms $\left[\left(\frac{\partial \mathbf{g}}{\partial \mathbf{x}}\right)^{T}\right]^{-1} \frac{\partial f}{\partial \mathbf{x}}$ and $\frac{\partial \mathbf{h}}{\partial \mathbf{x}}\left(\frac{\partial \mathbf{g}}{\partial \mathbf{x}}\right)^{-1}$ involved in (7) and (8) are the Lagrange multipliers vectors at the optimal solution corresponding to equality constraints (2) and to inequality constraints (3), respectively; hence they are provided as a by-product of the OPF computation [2]. While the former term is very useful and can be used directly in (7) to speed up sensitivities computation, the latter term is useless since only those components that correspond to binding inequality constraints are non-zero.

The incremental computational effort needed to derive the sensitivities in (7) and (8) is very small, since the power flow Jacobian $\frac{\partial \mathbf{g}}{\partial \mathbf{x}}$ is already available and factorized at the PF or OPF solution.

Next for each discrete variable $u_{d i}\left(\forall i=1, \ldots, n_{d}\right)$, one can therefore estimate linearly the change in the objective and the inequality constraints when moving $u_{d i}$ from its current discrete value $u_{d i}^{j_{i}}$ to its nearest lower value, $u_{d i}^{j_{i}-1}$ or to its nearest higher value, $u_{d i}^{j_{i}+1}$ :

$$
\begin{aligned}
\Delta f_{i}^{+} & =S_{u_{d i}}^{f}\left(u_{d i}^{j_{i}+1}-u_{d i}^{j_{i}}\right) & & \\
\Delta f_{i}^{-} & =S_{u_{d i}}^{f}\left(u_{d i}^{j_{i}-1}-u_{d i}^{j_{i}}\right) & & \\
\Delta h_{k i}^{+} & =S_{u_{d i}}^{h_{k}}\left(u_{d i}^{j_{i}+1}-u_{d i}^{j_{i}}\right), & & \forall k=1, \ldots, n_{h} \\
\Delta h_{k i}^{-} & =S_{u_{d i}}^{h_{k}}\left(u_{d i}^{j_{i}-1}-u_{d i}^{j_{i}}\right), & & \forall k=1, \ldots, n_{h}
\end{aligned}
$$

where $n_{h}$ is the dimension of vector $\mathbf{h}$ in (3).

Then we compute the value of the so-called merit function which combines the variation of the objective function and the variation of the degree of constraints violation:

$$
\begin{aligned}
& \eta_{i}^{+}=w_{f} \Delta f_{i}^{+}+\sum_{k=1}^{n_{h}} w_{h} \max \left[0, h_{k}\left(\mathbf{x}, \mathbf{u}_{c}, \mathbf{u}_{d}\right)+\Delta h_{k i}^{+}\right] \\
& \eta_{i}^{-}=w_{f} \Delta f_{i}^{-}+\sum_{k=1}^{n_{h}} w_{h} \max \left[0, h_{k}\left(\mathbf{x}, \mathbf{u}_{c}, \mathbf{u}_{d}\right)+\Delta h_{k i}^{-}\right]
\end{aligned}
$$

where $w_{f} \geq 0$ and $w_{h} \geq 0$ are weighting factors.

Observe that the terms related to inequality constraints contribute to the merit function only if some inequality constraints are violated after altering the value of discrete variable $u_{d i}$, i.e. if $h_{k}\left(\mathbf{x}, \mathbf{u}_{c}, \mathbf{u}_{d}\right)+\Delta h_{k i}^{+}>0$ or $h_{k}\left(\mathbf{x}, \mathbf{u}_{c}, \mathbf{u}_{d}\right)+\Delta h_{k i}^{-}>0$. The lower the value of the merit function the better the effect of moving the discrete variable.
Finally, the discrete variable $u_{d i}$ is moved to its nearest discrete value which leads to best overall improvement of optimality and feasibility, i.e.:

$$
\begin{array}{lll}
u_{d i}^{j_{i}} \leftarrow u_{d i}^{j_{i}+1} & \text { if } \quad \eta_{i}^{+} \leq \eta_{i}^{-} \text {or } \\
u_{d i}^{j_{i}} \leftarrow u_{d i}^{j_{i}-1} & \text { if } \quad \eta_{i}^{+}>\eta_{i}^{-}
\end{array}
$$

Notice that in the above procedure we have assumed that the discrete variables at the current iteration were already at one of their discrete values. However, depending on the procedure used to determine discrete variables, (e.g., starting with a continuous relaxation of the OPF, see below), this is generally not the case. In the situation where variable $u_{d i}^{j_{i}}$ does not have a discrete value, $u_{d i}^{j_{i}+1}$ and $u_{d i}^{j_{i}-1}$ from formulas (912) represent its nearest successive upper and lower discrete values.

On the other hand, if all discrete variables are already at discrete values, the value of a variable is actually modified only if it can significantly improve the merit function, i.e., if either $\eta_{i}^{+} \leq \eta_{t h}$ or $\eta_{i}^{-} \leq \eta_{t h}$. The value of this threshold $\eta_{t h}$ can be adapted in order to control the number of discrete variables allowed to move at a given iteration.

\section{Outline of the proposed algorithm}

The proposed iterative greedy algorithm is outlined as follows:

1) Solve the OPF continuous relaxation (discrete variables are treated as continuous).

2) At the current OPF optimum, compute the sensitivity of the objective function and inequality constraints with respect to discrete variables $\left(\mathbf{S}_{\mathbf{u}_{d}}^{f}\right.$ and $\mathbf{S}_{\mathbf{u}_{d}}^{\mathbf{h}}$ ) according to formulas (7) and (8).

3) For each discrete variable $u_{d i}\left(\forall i=1, \ldots, n_{d}\right)$, compute the merit function relative to its movement according to formulas (13) and (14).

- Update all discrete variables which have small enough merit function (given the threshold $\eta_{t h}$ ) or which are currently not yet at a discrete value (this will be the case only at the first iteration), by moving them to their nearest (upper or lower) discrete value, according to formulas (15) and (16). Let $\mathbf{u}_{d}^{\star}$ be the new values of discrete variables.

- If none of the discrete variable values has been changed with respect to the previous iteration, a near-optimal solution has been found and the computation terminates.

4) Run the OPF to optimize continuous variables only (discrete variables are frozen at their current value $\mathbf{u}_{d}^{\star}$ ).

- If the OPF objective value change with respect to either the previous iteration or the continuous relaxation OPF is smaller than a tolerance, then an acceptable near-optimal solution has been found and the computation terminates.

- Otherwise, go to step 2.

NB. To lighten the algorithm comprehension, the case where the OPF problem posed at step 4) is infeasible is discussed in Section III-D. 
Observe that, as soon as all discrete variables are at discrete values, the algorithm stops in one of the following two situations: (i) if for any discrete variable the merit function indicates that there is no significant impact by moving the variable to a new discrete value, or (ii) the OPF objective variation is deemed negligible.

Note that, unlike most methods existing in the literature, which search for the optimal value of discrete variables by considering only two discrete values for each one of them, the proposed approach allows the movement of discrete variables (one single discrete step at the time) to positions that best improve the optimality and feasibility. In other words, the proposed approach uses a larger discrete search space and hence has higher chances to find a feasible solution.

Although we formulated the algorithm by assuming that it searches from scratch, one may adapt it in order to take advantage of any other suitable approach for choosing an initial value of discrete variables. Thus, the proposed algorithm can start from any initial value of the discrete variables (e.g., stemming from a real-time operating point), and the sensitivity-based approach may be used as a post-processing of the round-off approach if the latter leads to an infeasible OPF or to an unacceptable deterioration of the objective.

One possible drawback of the proposed heuristic is related to the fact that it treats the different variables independently of each other and hence their cumulative effects on inequality constraints may not be properly taken into account. This holds true especially if discrete variables are related to devices which are located close to each other in the power system.

Hence, in order to take more explicitly into account the potential cumulative effect of the changes of several variable values we propose a simple heuristic technique which can replace the variable updating scheme at step 3) of the above algorithm:

1) rank discrete variables in increasing order of the merit function;

2) pick the top ranked discrete variable which has not been set yet to a discrete value and set it to its best discrete value;

3) update the inequality constraints (e.g., the bus voltage magnitudes) by considering linearly the effect of changing the value of the top ranked discrete variable;

4) if all discrete variables have been set to a discrete value stop, otherwise go to step 1.

Another possibility to take into account the cumulative effect and to overcome linear sensitivities limitation would consist in setting discrete variables to their discrete values by using a more progressive approach, e.g., by first updating only the most "efficient" ones instead of changing all of them at each iteration. In this case, the sensitivities of those discrete variables that have not been set yet would be updated by rerunning the OPF with the new values fixed for the remaining variables.

\section{Dealing with continuous OPF infeasibility}

As mentioned above, it is possible that during the iterative search of satisfactory configurations of discrete variables some of the configurations proposed by our algorithm lead to an OPF problem (1-4) which is infeasible.

We therefore propose to handle such situations by solving a minimum degree of constraints violation OPF problem instead of the original problem. More precisely, if for an established configuration of the discrete variables the OPF (1-4) which optimizes continuous variables only is infeasible, we solve instead the following OPF problem:

$$
\begin{aligned}
\min _{\mathbf{x}, \mathbf{u}_{c}, \mathbf{r}} & \mathbf{1}^{T} \mathbf{r} \\
\text { s.t. } & \mathbf{g}\left(\mathbf{x}, \mathbf{u}_{c}, \mathbf{u}_{d}\right)=\mathbf{0} \\
& \mathbf{h}\left(\mathbf{x}, \mathbf{u}_{c}, \mathbf{u}_{d}\right) \leq \mathbf{r} \\
& \underline{\mathbf{u}}_{c} \leq \mathbf{u}_{c} \leq \overline{\mathbf{u}}_{c} \\
& \mathbf{r} \geq \mathbf{0}
\end{aligned}
$$

in which positive relaxation variables (21) have been introduced in order to relax the inequality constraints (19). The objective (17) is the minimization of the degree of constraints violation in the sense of the $L_{1}$ norm.

The solution of problem (17-21) in the context of the proposed algorithm is very useful since it provides an optimum of the relaxed OPF which enables the derivation of sensitivities (7-8) and hence allows to carry on the algorithm. Incidentally, the solution of the problem (17-21) yields the degree of original continuous OPF problem infeasibility. This is a worthy information especially if the MINLP OPF (1-6) is infeasible whatever the values taken by discrete variables.

\section{NUMERICAL CASE STUDIES}

\section{A. Problem definition and test systems description}

We consider the OPF problem of minimizing the active power losses of the transmission system. We consider continuous control variables (e.g., slack generator active power, generators terminal voltage) and discrete control variables (e.g., controllable transformer ratios and shunt reactances). The equality constraints comprise the active and reactive power flows at all buses. The inequality constraints, on the other hand, concern branch current limits and bounds on voltage magnitudes, generator reactive powers, transformer ratios, and shunt reactance total capacities. Voltage magnitudes are allowed to vary between $0.95 \mathrm{pu}$ and $1.05 \mathrm{pu}$ at all buses. As regards discrete variables, we assume for all our test systems that the ratio of each tap-changer varies between $0.9 \mathrm{pu}$ and $1.1 \mathrm{pu}$ and has 21 discrete positions and therefore has a step of $0.01 \mathrm{pu}$, while the steps of the shunt compensation banks range from 20 to 40 Mvar.

We present detailed results obtained with the proposed approach in four test systems: a 60-bus system, which is a modified variant of the Nordic32 system [28], the IEEE300 bus system [29], a 618-bus system, and a modified ${ }^{1}$ planning model of the RTE (the French transmission system operator) system of 1203 buses. A summary of their characteristics is given in Table I. In this table $n, g, c, b, l, t, o, s, n_{c}$, and $n_{d}$ denote respectively the number of: buses, generators, loads,

\footnotetext{
${ }^{1}$ for the sake of dealing with a large number of discrete variables we have significantly increased the number of LTC transformers and shunt banks
} 
TABLE I

TEST SYSTEMS SUMMARY

\begin{tabular}{|c||c|c|c|c|c|c|c|c||c|c|}
\hline system & $n$ & $g$ & $c$ & $b$ & $l$ & $t$ & $o$ & $s$ & $n_{c}$ & $n_{d}$ \\
\hline \hline Nordic32 & 60 & 23 & 22 & 81 & 57 & 31 & 4 & 12 & 24 & 16 \\
\hline IEEE300 & 300 & 69 & 198 & 411 & 282 & 129 & 50 & 14 & 70 & 54 \\
\hline 618-bus & 618 & 72 & 352 & 1057 & 810 & 247 & 175 & 25 & 73 & 200 \\
\hline 1203-bus & 1203 & 177 & 767 & 1797 & 1394 & 403 & 203 & 36 & 178 & 239 \\
\hline
\end{tabular}

branches, lines, all transformers, transformers with controllable ratio, shunt elements, continuous control variables, and discrete control variables.

\section{B. Illustration of the proposed approach}

Let us illustrate how the proposed approach works by using the Nordic32 system. We first run the relaxed OPF (all variables are treated as continuous) and obtain a minimum power loss of 137.79 MW. Then we compute the sensitivities at the relaxed OPF optimum and, based on this information, we determine discrete values for all discrete variables. Then a new optimization of continuous control variables is performed. Now the active power loss is of $137.82 \mathrm{MW}$, i.e., very slightly higher than with the continuous solution. Since the obtained deterioration with respect to the continuous case is extremely small one can conclude that the convergence has been reached. As a matter of fact, checking the values of sensitivities at this optimal point reveals that there is no further benefit (i.e., no significant impact on the objective) to change any discrete variable to another discrete value.

Table II presents the values of active power loss obtained at the operating points computed on our four test systems by three different approaches: continuous relaxation (where all variables are treated as continuous), the classical round-off strategy, and the proposed sensitivity-based approach. Note that in all tests the sensitivity-based approach outperforms the round-off approach in terms of both objective quality, since it always leads to smaller losses, and robustness, since always leads to feasible solutions contrary to the round-off approach which leads to an infeasible continuous OPF for the IEEE300 system. On the other hand, the proposed sensitivity-based approach requires additional computational efforts with respect to the round-off technique, due to the sensitivities computation. This task is however extremely fast compared to the solution of the OPF itself.

One can also observe that, even when the number of discrete controls is rather large (e.g., for the 618-bus and 1203-bus systems), the discrete optimum reached is very close to that of the continuous approach. This effect is due to the rather small sensitivity of the objective function with respect to discrete controls changes for these test systems. As a consequence, the sensitivity-based approach has reached convergence in all cases in a single iteration.

\section{Analytical sensitivities validation by finite differences}

Table III shows the objective function change due to the movement of one discrete control at the time, determined by two approaches: finite differences based on a power flow computation and predictions by using our analytical sensitivities.
TABLE II

ACTIVE POWER LOSSES (MW) BY THREE APPROACHES

\begin{tabular}{|c||c|c|c|}
\hline system & continuous & round-off & sensitivity \\
\hline \hline Nordic32 & 137.79 & 137.92 & 137.82 \\
\hline IEEE300 & 386.60 & infeasible & 388.06 \\
\hline 618-bus & 844.25 & 846.46 & 845.41 \\
\hline 1203-bus & 2050.58 & 2054.15 & 2051.67 \\
\hline
\end{tabular}

TABLE III

OBJECTIVE FUNCTION CHANGE COMPUTED BY FINITE DIFFERENCES AND BY ANALYTICAL SENSITIVITIES FOR VARIOUS DISCRETE CONTROL CHANGES (ONE CONTROL VARIABLE CHANGE AT THE TIME): RESULTS USING THE 1203-BUS SYSTEM

\begin{tabular}{|c|c|c|c|c|c|c|c|c|c|}
\hline \multicolumn{7}{|c|}{ LTC number } & \multicolumn{5}{|c|}{ shunt number } \\
\hline 3 & 10 & 83 & 132 & 191 & 3 & 6 & 17 & 21 & 34 \\
\hline \hline \multicolumn{8}{|c|}{ real objective change (MW) by finite differences } \\
\hline 0.16 & 0.18 & -0.02 & 0.17 & 0.31 & -0.23 & -0.46 & -0.72 & -0.84 & -0.32 \\
-0.12 & -0.16 & 0.04 & -0.15 & -0.23 & 0.24 & 0.53 & 0.75 & 0.92 & 0.45 \\
\hline \multicolumn{8}{|c|}{ predicted objective change (MW) by analytical sensitivities } \\
\hline 0.16 & 0.20 & -0.06 & 0.18 & 0.37 & -0.24 & -0.52 & -0.74 & -0.89 & -0.39 \\
\hline
\end{tabular}

The approach by finite differences shows the objective shift when moving each control with one discrete step either up or down. The discrete step for any LTC ratio has been taken of $0.01 \mathrm{pu}$ and for any shunt of 30 MVar, respectively. For this comparison we use a non-optimized base case of the 1203-bus system.

We observe from Table III that the objective function change computed by both approaches is generally sufficiently close so as to validate the analytical sensitivities. Clearly small mismatches are expected due to the well known nonlinear local effect of reactive power flow. Observe also that for some controls (e.g., LTC 191 and shunt 34) increasing and decreasing with the same amount the current value of a discrete variable leads to rather different values. This mismatch is also attributable to the non-linearity introduced by generators which lose or regain their ability to control voltage subsequently to the action of other "reactive" power control devices (e.g., LTC ratio, shunt reactance, etc.). For instance, at the base case 15 generators have reached their maximum reactive power limit (these constraints are binding at the continuous OPF solution) but when injecting (resp. retrieving) some reactive power into the network, all these generators come back under voltage control (resp. a few other generators also lose their ability to control voltage).

Table IV provides on the other hand, for different pairs of weights $w_{f}$ and $w_{h}$, the objective function change and the overall bus voltage limit violations, obtained by the same two approaches, using the modified settings of the discrete controls suggested by our approach. The sensitivity-based approach estimates linearly the effect of simultaneously moving several control variables on the objective function and on the violated limits. The finite differences approach implements simultaneously the same changes of the values of the discrete variables as the sensitivity-based approach and, at the converged PF solution, computes the resulting variation of the objective function and the sum of all bus voltage limit violations.

Let us observe that there is a very good agreement among the two approaches concerning the overall bus voltage limit 
TABLE IV

OBJECTIVE FUNCTION AND OVERALL BUS VOLTAGE LIMIT VIOLATION CHANGES COMPUTED BY PF AND PREDICTED BY ANALYTICAL SENSITIVITIES FOR SIMULTANEOUS CONTROL MOVEMENTS DETERMINED BY USING VARIOUS VALUES OF WEIGHTS $w_{f}$ AND $w_{h}$ : RESULTS USING THE 1203-BUS SYSTEM

\begin{tabular}{|c|c|c|c|c|c|c|}
\hline$w_{f}>0$ & $w_{f}=1$ & $w_{f}=1$ & $w_{f}=1$ & $w_{f}=1$ & $w_{f}=1$ & $w_{f}=0$ \\
$w_{h}=0$ & $w_{h}=1$ & $w_{h}=10$ & $w_{h}=50$ & $w_{h}=100$ & $w_{h}=200$ & $w_{h}>0$ \\
\hline \hline \multicolumn{7}{|c|}{ real objective variation (MW) computed by PF } \\
\hline-19.78 & -19.77 & -17.08 & -13.93 & -10.01 & -7.24 & 0.47 \\
\hline \multicolumn{7}{|c|}{ predicted objective variation (MW) by analytical sensitivities } \\
\hline-27.83 & -27.73 & -23.76 & -20.28 & -15.35 & -11.98 & 0.10 \\
\hline \hline \multicolumn{7}{|c|}{ real overall bus voltage limits violation (pu) computed by PF } \\
\hline 1.381 & 1.145 & 0.266 & 0.083 & 0.028 & 0.008 & 0.000 \\
\hline predicted overall bus voltage limits violation (pu) by analytical sensitivities \\
\hline 1.221 & 1.028 & 0.255 & 0.082 & 0.028 & 0.008 & 0.000 \\
\hline
\end{tabular}

violations. On the other hand, the sensitivity-based approach tends to overestimate the change of the objective function by about $40-50 \%$. Since the sensitivities have been validated when moving controls one at the time, this mismatch is attributable to the large number of discrete variables which move (around 200 in average) and, to a lesser extent, to the fact that the slack generator compensating the losses variation is located outside the optimized system. In these conditions the mismatch between sensitivity-based and finite differences approaches seems reasonable.

Note also that, at the PF solution obtained after discrete variables changes, the larger the weight relative to the objective function, the larger the objective improvement but the higher the overall voltage limit violations.

\section{Sensitivity of the OPF result with respect to the weights on objective and violated inequality constraints}

We now provide some results on the sensitivity of the OPF output with respect to the weights of the objective function and violated inequality constraints, $w_{f}$ and $w_{h}$, involved in the merit function formulas (13) and (14). Without loss of generality we use the 1203-bus system for this example and consider as base case the OPF continuous relaxation optimum.

Let us first explain how the meaning of these pairs of weights can be interpreted. For instance the pair $w_{f}=1$ and $w_{h}=10$ means that a discrete variable is allowed to move to a new discrete position if this leads to an overall voltage limit violation of $0.01 \mathrm{pu}$ and the active power losses diminish by more than 0.1 MW, and if the merit function corresponding to this movement is smaller than its value corresponding to the movement of the variable in the opposite direction.

Table $\mathrm{V}$ provides, for different pairs of weights $w_{f}$ and $w_{h}$, the active power losses at the OPF solution and the active power losses and the sum of bus voltage limit violations at the PF solution obtained after the update of discrete values.

We first notice that the optimum value of power losses is rather stable over a quite large range of weight values, i.e. $w_{f}=1$ and $w_{h} \in\left[\begin{array}{ll}50 & 200\end{array}\right]$, which shows the robustness of the proposed approach. Furthermore, the value of active losses obtained with the proposed approach is smaller than with the round-off approach (see Table II) for an even larger range of weights. The results show also that putting a too
TABLE V

SENSITIVITY OF ACTIVE POWER LOSSES WITH RESPECT TO THE CHOICE OF WEIGHTS $w_{f}$ AND $w_{h}$ : RESULTS USING THE 1203-BUS SYSTEM

\begin{tabular}{|l|c|c|c|c|c|c|}
\hline$w_{f}>0$ & $w_{f}=1$ & $w_{f}=1$ & $w_{f}=1$ & $w_{f}=1$ & $w_{f}=1$ & $w_{f}=0$ \\
$w_{h}=0$ & $w_{h}=1$ & $w_{h}=10$ & $w_{h}=50$ & $w_{h}=100$ & $w_{h}=200$ & $w_{h}>0$ \\
\hline \hline \multicolumn{7}{|c|}{ active power losses (MW) at the OPF solution } \\
\hline 2066.17 & 2065.75 & 2055.03 & 2051.75 & 2051.67 & 2051.65 & 2052.26 \\
\hline \hline \multicolumn{7}{|c|}{ active power losses (MW) at the PF solution } \\
\hline 2043.65 & 2043.76 & 2048.11 & 2051.00 & 2051.69 & 2052.13 & 2053.60 \\
\hline \hline \multicolumn{7}{|c|}{ sum of bus voltage limit violations at the PF solution (pu) } \\
\hline 1.955 & 1.867 & 0.171 & 0.018 & 0.010 & 0.005 & 0.003 \\
\hline
\end{tabular}

strong emphasis on the objective function improvement (e.g., the extreme case where $w_{f}>0$ and $w_{h}=0$, and where $w_{f}=w_{h}=1$ ) leads actually to a rather poor value of the objective function. In contrast, the extreme case where the impact of discretization on the objective function is not at all taken into account $\left(w_{f}=0\right.$ and $\left.w_{h}>0\right)$ leads nevertheless to a rather good value of the power losses, although it is slightly suboptimal.

Finally we observe that, at the PF solution obtained after discrete variables are updated, the larger the weight relative to the objective function improvement, the smaller the active power losses and the higher the overall voltage limit violations.

\section{E. Starting from different discrete variables settings}

We now use the 1203-bus system and initialize the discrete variables in the sensitivity-based approach by means of the round-off approach applied at the continuous OPF relaxation optimum. Then we apply the proposed approach and the merit functions indicate that 80 discrete controls (out of 239) should be moved. We move them to their suggested values and next we re-run the OPF with continuous variables only and observe that it leads to a losses value of $2053.20 \mathrm{MW}$ of losses, i.e., 0.95 MW less than with the round-off approach (see Table II). The analysis of the merit functions at this OPF solution indicates that no major benefit would be expected by changing the discrete variables values and hence the computations terminate. Thus, when using this initialization, although the losses are improved with $0.95 \mathrm{MW}$ with respect to the round-off approach, they are $1.53 \mathrm{MW}$ higher than when using first a continuous OPF relaxation (see Table II). Furthermore, we have observed that even by changing the relative values of weights $w_{f}$ and $w_{h}$, the last sub-optimum (2053.20 MW) is not improved significantly. We conclude that when discrete variables approach very closely their optimum values the information provided by the merit function may be less efficient.

Let us now start from a non-optimized base case of the 1203-bus system and with given values for discrete controls. Figure 1 plots the evolution of active power losses when using this starting point in the sensitivity-based approach. One can observe that the sensitivity-based procedure takes 4 iterations to converge and that the losses decrease is less and less significant with the increase of the iteration number. However, the final value of losses (2056.30 MW) is slightly larger than with the round-off approach (i.e., 2054.15 MW) and even more suboptimal with respect to the case reported in Table II where 


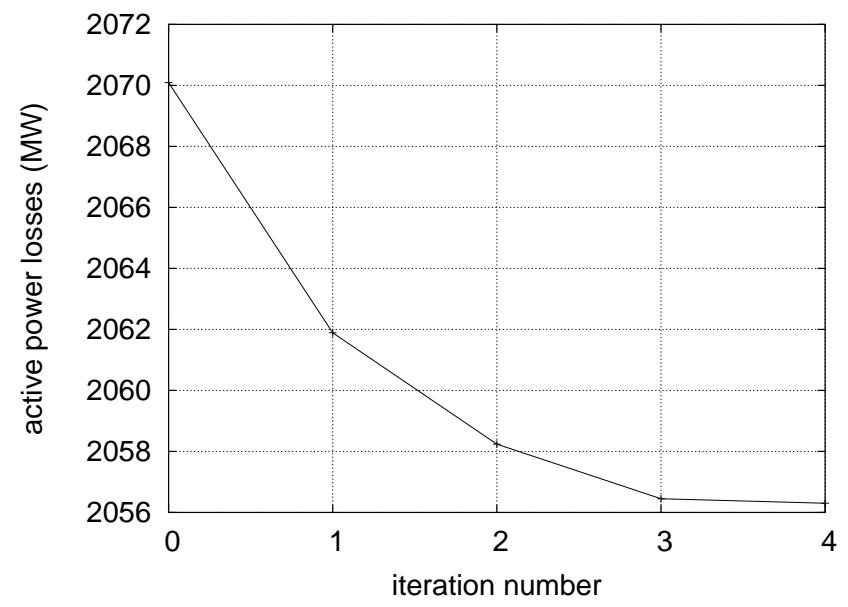

Fig. 1. Evolution of active power losses when starting from a non-optimized base case: results obtained using the 1203-bus system

the discrete variables are initialized by running the continuous relaxation OPF version (i.e., 2051.67 MW). An analysis of the converged solution of the sensitivity-based procedure reveals that the two initializations (continuous relaxation and nonoptimized base case) have led to different settings for most discrete variables. One can conclude that depending on the starting point the sensitivity-based method may be "trapped" in a less interesting near-optimum, or the number of iterations to "unblock" the method may be very large due to fact that the discrete variables are allowed to move only one step at the time so as to preserve the validity of sensitivities.

On the other hand, in contrast with the round-off approach, the sensitivity-based approach has the ability to improve losses for a given operation point (stemming for instance from realtime) and to easily limit the number of controls allowed to move so as to improve the objective.

These two examples clearly show that it is better to initialize discrete variables starting from a relaxed OPF solution.

\section{F. Dealing with infeasible discrete variables configurations}

We now show how the proposed approach copes with infeasible situations which appear owing to inadequate discrete variable settings, but for which the original MINLP OPF problem is feasible. We illustrate such a case on the IEEE300 system. The active power losses at the relaxed OPF solution are of $386.60 \mathrm{MW}$ (see Table II). Then, after rounding-off the discrete variables, we notice that the subsequent OPF that optimizes continuous variables is infeasible. In contrast, after setting discrete variables by the sensitivity approach, the subsequent OPF converges to a solution where the power losses are of $388.06 \mathrm{MW}$, i.e., slightly larger than in the relaxed approach. This illustrates the fact that in some cases the simple heuristic behind the round-off approach may lead to infeasible discrete variable configurations, even if the original MINLP problem is feasible.

Now we show how our approach can provide a solution starting from an infeasible configuration of discrete variables. To this end, we first initialize discrete variables to the values provided by the round-off approach. Then we solve the minimum infeasibility degree problem (17-21) which provides a feasible solution where two minimum voltage limits have been relaxed with $0.02 \mathrm{pu}$ and $0.01 \mathrm{pu}$, respectively. Next sensitivities are derived at this optimum and discrete variables are moved to new discrete values according to the rules of the sensitivity-based approach. Finally, by re-running the OPF with continuous variables only the problem is feasible and the losses are of $389.79 \mathrm{MW}$.

\section{Conclusions}

This paper has proposed a new heuristic approach to deal with discrete variables in an OPF. The proposed approach relies on first order sensitivities of the objective function and of the inequality constraints with respect to discrete variables, that may be computed efficiently at the solution of a PF or an OPF software. In our approach, these sensitivities are combined in a merit function to determine the direction of movement of a discrete variable. An iterative algorithm is proposed to greedily move the discrete variables to their nearest upper or lower bound at each iteration. This algorithm may be started from scratch, or it may be used as a postprocessor to other methods, so as to improve the value of the objective function or to find a feasible configuration of the discrete variables. Nevertheless, much better results in terms of objective quality and computational speed have been obtained when starting from a continuous relaxation OPF solution.

The validation of the approach on four test system has shown that the proposed sensitivity-based approach outperforms the classical round-off approach for all test systems in terms of both numerical robustness and objective quality. The main features of the sensitivity-based approach are: (i) it can pass through infeasible points, using them just as a basis to enable sensitivities computation and hence carrying on the procedure, (ii) it can start from any configuration of the discrete variables values, and (iii) it restricts at each iteration the movement of each discrete variable to one of its neighbouring discrete positions, so as to preserve the validity of sensitivity information.

A slight drawback of the method is that it depends on the choice of the weights relative to the objective improvement and violated inequality constraints, involved in the merit functions. However, the numerical results have shown that the quality of the final optimum is very good for a rather large range of these weights.

The proposed approach leads to an increase of the computational effort with respect to the round-off technique. However, this increase is most of the times negligible.

One direction of future work is to build a MILP problem in order to set the discrete variables instead of the heuristic technique used in the paper. The MILP will be fed with the sensitivity of the objective and inequality constraints with respect to discrete variables changes.

Another direction of work is to compare whether computing sensitivities by means of an OPF program would provide better results than the sensitivities derived at the power flow solution. 


\section{ACKNOWLEDGMENTS}

We thank RTE (the French transmission system operator) for allowing us to use and publish results with their data.

This paper presents research results of the Belgian Network DYSCO, funded by the Interuniversity Attraction Poles Programme, initiated by the Belgian State, Science Policy Office. Florin Capitanescu and Louis Wehenkel acknowledge their funding by the FP7 EC project PEGASE. The scientific responsibility rests with the authors.

\section{REFERENCES}

[1] J. Carpentier, "Contribution à l'étude du dispatching économique", Bulletin de la Societé Française d'Electricité, vol. 3, 1962, pp. 431-447.

[2] H.W. Dommel and W.F. Tinney, "Optimal power flow solutions", IEEE Trans. PAS, vol. PAS-87, no. 10, October 1968, pp. 1866-1876.

[3] O.K. Gupta and A. Ravindran, "Branch and Bound Experiments in Convex Nonlinear Integer Programming", Manage Sci., vol. 31, no. 12, 1985, pp. 1533-1546.

[4] I. Quesada and I.E. Grossmann, "An LP/NLP Based Branch and Bound Algorithm for Convex MINLP Optimization Problems", Computers Chem. Eng., vol. 16, no. 10/11, 1992, 937-947.

[5] M.A. Duran and I.E. Grossmann, "An Outer-Approximation Algorithm for a Class of Mixed-integer Nonlinear Programs", Math. Program., vol. 36, 1986, pp. 307-339.

[6] R. Fletcher and S. Leyffer, "Solving Mixed Integer Programs by Outer Approximation", Math. Program., vol. 66, 1994, pp. 327-349.

[7] J.F. Benders, "Partitioning procedure for solving mixed variables programming problems", Numerische Mathematics, vol. 4, 1962, pp. 238252.

[8] A.M. Geoffrion, "Generalized Benders decomposition", Journal of Optimization Theory and Applications, vol. 10, no. 4, 1972, pp. 237-260.

[9] J.E. Mitchell and M.J. Todd, "Solving combinatorial optimization problems using Karmarkars algorithm", Mathematical Programming, vol. 56, no. 1-3, pp. 245-284, Aug. 1992.

[10] T. Westerlund and F. Petersson, "A Cutting Plane Method for Solving Convex MINLP Problems", Computers Chem. Eng., vol. 19, 1995, pp. 131-136.

[11] B. Stott, O. Alsac, and A.J. Monticelli, "Security analysis and optimization" (Invited Paper), IEEE Proc., vol. 75, no. 12, December 1987, pp. 1623-1644.

[12] W.F. Tinney, J.M. Bright, K.D. Demaree, and B.A. Hughes, "Some deficiencies in Optimal Power Flow”, IEEE Trans. on Power Syst., vol. 3, 1988, pp. 676-683.
[13] J.A. Momoh, R.J. Koessler, M.S. Bond, B. Stott, D. Sun, A. Papalexopoulos, and P. Ristanovic, "Challenges to optimal power flow", IEEE Trans. Power Syst. Vol. 12, No. 1, February 1997, pp. 444-455.

[14] A.D. Papalexopoulos, C.F. Imparato, and F.W. Wu, "Large-Scale Optimal Power Flow: Effects of Initialization, Decoupling \& Discretization", IEEE Trans. on Power Syst., vol. 4, 1989, pp. 748-759.

[15] K. Karoui, L. Platbrood, H. Crisciu, and R.A. Waltz, "New large-scale security constrained optimal power flow program using a new interiorpoint algorithm", 5th International Conference on European Electricity Market, May 2008.

[16] E. Liu, A.D. Papalexopoulos, and W.F. Tinney, "Discrete Shunt controls in a Newton Optimal Power Flow", IEEE Trans. on Power Syst., vol. 7, 1992, pp. 1519-1528.

[17] M. Liu, S.K. Tso, and Y. Cheng, "An Extended Nonlinear Primal-Dual Interior-Point Algorithm for Reactive Power Optimization of Large-Scale Power Systems with Discrete Control Variables", IEEE Trans. on Power Syst., vol. 17, no. 4, 2002, pp. 982-991.

[18] A.G. Bakirtzis and A.P. Meliopoulos, "Incorporation of switching operations in power system corrective control computations", IEEE Trans. on Power Syst., vol. 2, August 1987, pp. 669-676.

[19] S.Y. Lin, Y.C. Ho, and C.H. Lin, "An Ordinal Optimization TheoryBased Algorithm for Solving Optimal Power Flow Problem with Discrete Control Variables", IEEE Trans. on Power Syst., vol. 19, 2004, pp. 276286

[20] K. Aoki, M. Fan, and A. Nishikori, "Optimal var planning by approximation method for recursive mixed-integer linear programming", IEEE Trans. on Power Syst., vol. 3, November 1988, pp. 1741-1747.

[21] X. Ding, X. Wang, and Y.H. Song, "Interior point cutting plane method for optimal power flow", IMA Journal of management mathematics, vol. 15, no. 4, 2004, pp. 355-368.

[22] L.L. Lai, J.T. Ma, R. Yokoyama, and M. Zhao, "Improved Genetic Algorithms for Optimal Power Flow under both normal and contingent operation states", Journal of Electric Power and Energy Systems, vol. 19, no. 5,1997 , pp. 287-292.

[23] A.G. Bakirtzis, P.N. Biskas, C.E. Zoumas, and V. Petridis, "Optimal Power Flow by Enhanced Genetic Algorithm", IEEE Trans. on Power Syst., vol. 17, 2002, pp. 229-236.

[24] L. Chen, H. Suzuki, and K. Katou, "Mean-field theory for optimal power flow”, IEEE Trans. on Power Syst., vol. 12, 1997, pp. 1481-1486.

[25] T. Kulworawanichpong and S. Sujitjorn, "Optimal Power Flow using tabu search", IEEE Power Engineering review, 2002, pp. 37-40.

[26] W. Yan, F. Liu, C.Y. Chung, and K.P. Wong, "A Hybrid Genetic Algorithm-Interior Point Method for Optimal Reactive Power Flow", IEEE Trans. on Power Syst., vol. 21, no. 3, August 2006, pp. 1163-1169.

[27] P.R. Gribik, D. Shirmohammadi, S. Hao, and C.L. Thomas, "Optimal Power Flow sensitivity analysis", IEEE Trans. on Power Syst., vol. 5, no. 3, August 1990, pp. 969-978.

[28] CIGRE Task Force 38.02.08, "Long-Term Dynamics, Phase II", 1995.

[29] IEEE300 bus system, available online at http://www.ee.washington.edu, 1996 